\title{
Cambios en el pelaje del roedor subterráneo Ctenomys talarum: posible mecanismo térmico compensatorio
}

\author{
Fur changes in the subterranean rodent Ctenomys talarum: \\ possible thermal compensatory mechanism \\ ANA P. CUTRERA ${ }^{1,2} \&$ CARLOS D. ANTINUCHI ${ }^{2}$ \\ ${ }^{1}$ Consejo Nacional de Investigaciones Científicas y Técnicas (CONICET), Argentina; \\ e-mail: acutrera@mdp.edu.ar \\ ${ }^{2}$ Laboratorio de Ecofisiología, Universidad Nacional de Mar del Plata, Argentina
}

\begin{abstract}
RESUMEN
En este trabajo se evaluaron los cambios en densidad y longitud del pelaje dorsal y ventral en el roedor subterráneo Ctenomys talarum, como posible mecanismo compensatorio frente a cambios de temperatura estacionales en el ambiente de la cueva, en especial durante la época estival, y durante el período de preñez en las hembras, ya que ambas etapas representan desafíos para la termorregulación en esta especie. Se observó que el pelaje ventral fue significativamente más corto y menos denso que el dorsal en machos, hembras preñadas y hembras no preñadas en las dos estaciones evaluadas. La longitud del pelaje tanto dorsal como ventral en los tres grupos fue significativamente menor durante la estación de temperaturas más cálidas. En la estación cálida, las hembras preñadas exhibieron un pelaje ventral significativamente más corto que el pelaje ventral de machos y de hembras no preñadas. Se discuten las posibles ventajas térmicas que podrían representar las modificaciones observadas en las características del pelaje de esta especie, con énfasis en las restricciones que impone el ambiente subterráneo en cuanto a los mecanismos disponibles de disipación del calor corporal.
\end{abstract}

Palabras clave: pelaje, ambiente subterráneo, Ctenomys talarum, disipación.

\begin{abstract}
In this work, the changes in fur density and length in the subterranean rodent Ctenomys talarum were evaluated as a possible compensatory mechanism during seasonal temperature changes in their burrow environment and during pregnancy in females, both situations being thermoregulatory challenging in this species. The ventral fur was shorter and less dense than the dorsal fur in the three groups (males, nonpregnant females and pregnant females) and in the two seasons evaluated. Ventral and dorsal furs were significantly shorter during the warm seasons in the three groups. In the warm season, pregnant females had a ventral fur significantly shorter than that of males and non-pregnant females. The possible thermal advantages that the observed fur changes might represent for the species are discussed, with emphasis on the constraints imposed by the subterranean environment on available ways of dissipating body heat.
\end{abstract}

Key words: fur, subterranean environment, Ctenomys talarum, dissipation.

\section{INTRODUCCIÓN}

El ambiente subterráneo, aunque estructuralmente simple, es físicamente desafiante, particularmente en aspectos relacionados con la locomoción y la termorregulación de los organismos que lo habitan (Klauer et al. 1997). Las cuevas se encuentran pobremente ventiladas y su atmósfera es cálida y saturada de humedad (McNab 1966). Por lo tanto, aunque este microclima relativamente estable reduce la necesidad de mecanismos efectivos de producción y almacenamiento de calor, a su vez agudiza el problema de la disipación del calor corporal y del sobrecalentamiento (Mc Nab 1979, Klauer et al. 1997, Buffenstein 2000). En particular, en los roedores subterráneos la pérdida de calor por medio de la radiación es escasa, la transpiración es reducida debido a la alta humedad relativa en el ambiente de la cueva y la convección se encuentra impedida debido a la baja circulación de aire en este ambiente (Buffenstein 2000), por lo que la conducción se vuelve la principal vía de pérdida de calor en estos roedores ( $\mathrm{McNab}$ 1966, 1979). 
La pérdida de calor por conducción depende de las dimensiones del área de intercambio y de las propiedades conductivas de las superficies que se hallan en contacto (Cossins \& Bowler 1987). Por consiguiente, el rol del pelaje es esencial en este mecanismo. En los roedores subterráneos, las características estructurales del pelaje han sido estudiadas principalmente en Spalax ehrenbergi y Heterocephalus glaber (Klauer et al. 1997), con énfasis en su función protectora durante el cavado y en su rol en la percepción táctil del ambiente. Por otro lado, la función críptica del pelaje fue estudiada en el pocket gopher Geomys bursarius (Krupa \& Geluso 2000). Sin embargo, aunque es obvia su función como aislante térmico, se ha destinado poca atención a sus propiedades termorregulatorias en este grupo de roedores. En cambio, los estudios sobre ajustes compensatorios en la densidad y longitud del pelaje y sus efectos sobre la conductancia térmica se han concentrado en los roedores de superficie, detectándose marcadas diferencias entre las distintas estaciones del año (Hart \& Heroux 1953, Gorecki 1966, Khateeb \& Johnson 1971, Sealander 1972, Bolshakov 1984, Bozinovic et al. 1990, Bozinovic \& Merrit 1992).

No solo los aspectos relacionados con la plasticidad estacional de ciertos componentes de la energética de los roedores subterráneos han sido escasamente estudiados, sino también aquellos que tratan sobre los ajustes que se producen durante períodos de la vida de estos animales que resultan estresantes desde el punto de vista termorregulatorio, como lo constituye la preñez y la lactancia en las hembras. Estas experimentan cambios en forma, tamaño, peso, patrones de vascularización y tasa metabólica de reposo que conducen a modificaciones en las propiedades de transferencia de calor, las cuales deberían encontrarse, según Buffenstein (2000), en equilibrio con las condiciones físicas del interior de la cueva.

El roedor subterráneo sudamericano Ctenomys talarum representa un modelo interesante para evaluar los cambios de pelaje como posible mecanismo compensatorio frente a los cambios de temperatura estacionales en el ambiente de la cueva, en especial durante la época estival, y durante el período de preñez en las hembras (cuyo peso corporal se incrementa en hasta un $41 \%$ durante la gestación, ver Zenuto et al. 2002a), ya que ambas etapas representan desafíos para la termorregulación en esta especie.

En este estudio, se predice que: (1) la densidad y longitud del pelaje en esta especie se reducen durante las estaciones donde se registran las mayores temperaturas ambientales en las cuevas.

(2) En particular para las hembras, que experimentan también una reducción en dichas características del pelaje durante el período de preñez, con respecto a las hembras no preñadas y machos.

(3) Por último, en todos los animales dicha reducción es más acentuada en el vientre, en comparación con el pelaje del dorso, debido a que es la superficie ventral la que se encuentra en mayor contacto con el suelo y a través de la cual la disipación de calor corporal por conducción sería mayor.

\section{MATERIALES Y MÉTODOS}

Los animales utilizados en el estudio $(n=57)$ fueron capturados estacionalmente en la zona de Mar de Cobo (37²45' S, 57ํ5' O), Provincia de Buenos Aires, Argentina, con trampas de captura viva tipo tubo, desde diciembre de 2000 hasta septiembre de 2001. En la cueva de cada individuo capturado se tomó un registro de la temperatura del suelo fuera de la cueva (a $10 \mathrm{~cm}$ de la entrada de esta) y dentro de la misma (registrada a $20 \mathrm{~cm}$ de la zona de apertura realizada para colocar la trampa, inmediatamente luego de abrir la cueva) con un termómetro electrónico (Cole Parmer modelo 8402-10). Con este instrumento también se registró la temperatura del aire de superficie (registrada en la superficie externa de la cueva, cercana a la entrada de la misma) y del aire a $50 \mathrm{~cm}$ del suelo.

Los animales fueron pesados inmediatamente después de ser capturados y fueron sacrificados mediante una alta dosis de anestesia. La determinación de la condición reproductiva de las hembras se realizó mediante la observación de los cuernos uterinos, su forma y grado de irrigación, como así también se detectó la presencia o no de embriones. El estado de adultez en los machos se estableció mediante la observación de un frotis de epidídimo, analizando la abundancia de células espermáticas al microscopio (45x). Solo los machos y hembras maduros sexualmente fueron empleados en este estudio, ya que los juveniles presentan características especiales del pelaje, siendo este una combinación del pelaje de cría y de adulto (ver Zenuto et al. 2002a).

Se obtuvo una muestra de pelaje de $4 \mathrm{~mm}^{2}$ del dorso y del vientre de machos ( $\mathrm{n}$ fría $=12$, $\mathrm{n}$ cálida $=11)$ y hembras adultas, estas últimas tanto preñadas ( $\mathrm{n}$ fría $=7, \mathrm{n}$ cálida $=8$ ) como no preñadas ( $\mathrm{n}$ fría $=8, \mathrm{n}$ cálida $=11$ ). El con- 
teo de pelaje fue realizado bajo lupa binocular $(6,4 \mathrm{x})$ y con un ocular graduado se midió la longitud de tres pelos extraídos al azar de cada muestra de pelaje dorsal y ventral obtenida de cada individuo analizado. Los resultados son reportados como valor promedio \pm desviación estándar.

\section{Análisis estadísticos}

Las medias de temperaturas del aire de superficie, del aire a $50 \mathrm{~cm}$ de la superficie y del suelo dentro y fuera de la cueva fueron comparadas entre estaciones por medio de una prueba de ANOVA (STATISTICA® 1999, Kernel Release 5.5, Stat Soft Inc.). Para las comparaciones a posteriori se empleó la prueba de Tukey para muestras de $\mathrm{n}$ desigual. Con el fin de evaluar si el peso de los individuos tenía efecto sobre la densidad y longitud del pelaje, se utilizó un análisis de regresión simple para cada estación. Se empleó una prueba de ANOVA de tres vías con un factor con medidas repetidas para evaluar el efecto del sexo/condición reproductiva de las hembras, la estación del año y la región corporal sobre la densidad del pelaje. Se aplicó la misma prueba estadística para evaluar el efecto de estos factores sobre la longitud del pelaje. Para evaluar los efectos significativos de factores que no habían sido contemplados en las predicciones iniciales (i.e., interacciones), se utilizó a posteriori la prueba de Tukey para muestras de $\mathrm{n}$ desigual (STATISTICA ${ }^{\circledR}$ 1999, Kernel Release 5.5, Stat Soft Inc.).

\section{RESULTADOS}

\section{Ambiente térmico dentro y fuera de las cuevas}

La temperatura del aire de superficie, del aire a $50 \mathrm{~cm}$ del suelo y del suelo dentro y fuera de las cuevas de $C$. talarum difirieron de acuerdo al mes del año en que fueron registradas (Tabla 1; ANOVA; $\mathrm{F}_{3,53}=46,35-87,22 ; \mathrm{P}<0,001$ para todas las estaciones). Para dichas temperaturas, se observaron diferencias significativas entre los meses de diciembre y junio, diciembre y septiembre, marzo y junio, y marzo y septiembre (Tukey para muestras de $\mathrm{n}$ desigual, $\mathrm{P}<$ $0,0001)$, siendo mayores las temperaturas de diciembre y marzo. Sin embargo, no se registraron diferencias significativas entre los meses de diciembre y marzo ni entre junio y septiembre (Tukey para muestras de $\mathrm{n}$ desigual, $\mathrm{P}>0,05$ ). Por lo tanto, para los análisis de ANOVA posteriores, se emplearon dos estaciones: cálida (diciembre y marzo) y fría (junio y septiembre).

\section{TABLA 1}

Temperaturas del aire de superficie (T aire superficie), del aire a $50 \mathrm{~cm}$ del suelo $(\mathrm{T} 50 \mathrm{~cm})$, del suelo dentro de la cueva ( $\mathrm{T}$ suelo dentro) y del mismo fuera de la cueva (T suelo fuera) registradas durante los meses de diciembre, marzo, junio y septiembre. Letras diferentes indican diferencias significativas entre meses. Los resultados se expresan como valor medio \pm desviación estándar

Temperatures of the surface air ( $\mathrm{T}$ aire superficie), of the air at $50 \mathrm{~cm}$ from the surface $(\mathrm{T} 50 \mathrm{~cm})$, of the soil inside the burrow (T suelo dentro) and the soil outside the burrow (T suelo fuera) recorded during the months of December, March, June and September. Different letters represent significant differences among months. Results are reported as average \pm standard deviation

\begin{tabular}{lllll}
\hline Mes & $\begin{array}{l}\mathrm{T}^{\circ} \text { aire superficie } \\
\left({ }^{\circ} \mathrm{C}\right)\end{array}$ & $\begin{array}{l}\mathrm{T}^{\circ} 50 \mathrm{~cm} \\
\left({ }^{\circ} \mathrm{C}\right)\end{array}$ & $\begin{array}{l}\mathrm{T}^{\circ} \text { suelo dentro } \\
\left({ }^{\circ} \mathrm{C}\right)\end{array}$ & $\begin{array}{l}\mathrm{T}^{\circ} \text { suelo fuera } \\
\left({ }^{\circ} \mathrm{C}\right)\end{array}$ \\
\hline $\begin{array}{l}\text { Diciembre } \\
(\mathrm{n}=15)\end{array}$ & $29,08 \pm 2,66 \mathrm{a}$ & $26,18 \pm 2,6 \mathrm{a}$ & $22,6 \pm 1,48 \mathrm{a}$ & $26,16 \pm 3,06 \mathrm{a}$ \\
$\begin{array}{l}\text { Marzo } \\
(\mathrm{n}=15)\end{array}$ & $26,32 \pm 2,54 \mathrm{a}$ & $23,61 \pm 3,56 \mathrm{a}$ & $21,55 \pm 1,04 \mathrm{a}$ & $23,04 \pm 2,1 \mathrm{a}$ \\
$\begin{array}{l}\text { Junio } \\
(\mathrm{n}=13)\end{array}$ & $9,54 \pm 2,93 \mathrm{~b}$ & $8,85 \pm 1,75 \mathrm{~b}$ & $12,94 \pm 2,16 \mathrm{~b}$ & $12,23 \pm 3,85 \mathrm{~b}$ \\
$\begin{array}{l}\text { Septiembre } \\
(\mathrm{n}=14)\end{array}$ & $9,09 \pm 5,1 \mathrm{~b}$ & $9,93 \pm 3,38 \mathrm{~b}$ & $11,3 \pm 1,22 \mathrm{~b}$ & $12,47 \pm 2,26 \mathrm{~b}$ \\
& & & & \\
\hline
\end{tabular}




\section{Densidad del pelaje}

No se encontró relación entre el peso de los individuos y la densidad del pelaje dorsal en la estación fría $\left(\mathrm{n}=27 ; \mathrm{r}^{2}=0,06 ; \mathrm{F}_{1,25}=1,45 ; \mathrm{P}=\right.$ $0,24)$ ni en la cálida $\left(n=30 ; r^{2}=0,016 ; F_{1,28}=\right.$ $0,45 ; \mathrm{P}=0,51)$. Tampoco se encontró relación entre el peso de los individuos y la densidad del pelaje ventral en la estación fría $\left(n=27 ; r^{2}=\right.$ 0,$\left.08 ; \mathrm{F}_{1,25}=2,1 ; \mathrm{P}=0,16\right)$ ni la cálida $(\mathrm{n}=30$; $\left.\mathrm{r}^{2}=0,0008 ; \mathrm{F}_{1,28}=0,023 ; \mathrm{P}=0,88\right)$. Se observó efecto de la región corporal sobre la densidad del pelaje mientras que el efecto de la estación resultó marginalmente significativo (Tabla 2, Fig. 1). En este sentido, tanto los machos como las hembras preñadas y no preñadas presentaron un pelaje dorsal significativamente más denso que el ventral en ambas estaciones.

TABLA 2

Tabla de ANOVA de tres vías con el sexo/condición reproductiva de las hembras (machos, hembras preñadas y hembras no preñadas, 1) y la estación del año (cálida y fría, 2) como los factores "entre individuos" y la región corporal (dorso y vientre, 3) como el factor "en individuos" para evaluar su efecto el sobre la densidad del pelaje

Table of a three-way ANOVA, with sex/reproductive condition of the females (males, pregnant females and non-pregnant females, 1) and season of the year (warm and cold, 2) as between-subject effects and body region (back and belly, 3) as within- subject effect to evaluate their effect on fur density

\begin{tabular}{|c|c|c|c|c|}
\hline Factor & Grados de libertad & Cuadrado medio & Valor de F & Valor de P \\
\hline 1 & 2 & 158698 & 2,003 & 0,145 \\
\hline 2 & 1 & 316132 & 3,99 & 0,051 \\
\hline 12 & 2 & 50127 & 0,633 & 0,535 \\
\hline Error & 51 & 79223 & & \\
\hline 3 & 1 & 7704044 & 192,75 & $<0,0001$ \\
\hline 31 & 2 & 72975 & 1,826 & 0,183 \\
\hline 32 & 1 & 77401 & 1,937 & 0,155 \\
\hline 123 & 2 & 39574 & 0,99 & 0,379 \\
\hline Error & 51 & 39969 & & \\
\hline
\end{tabular}

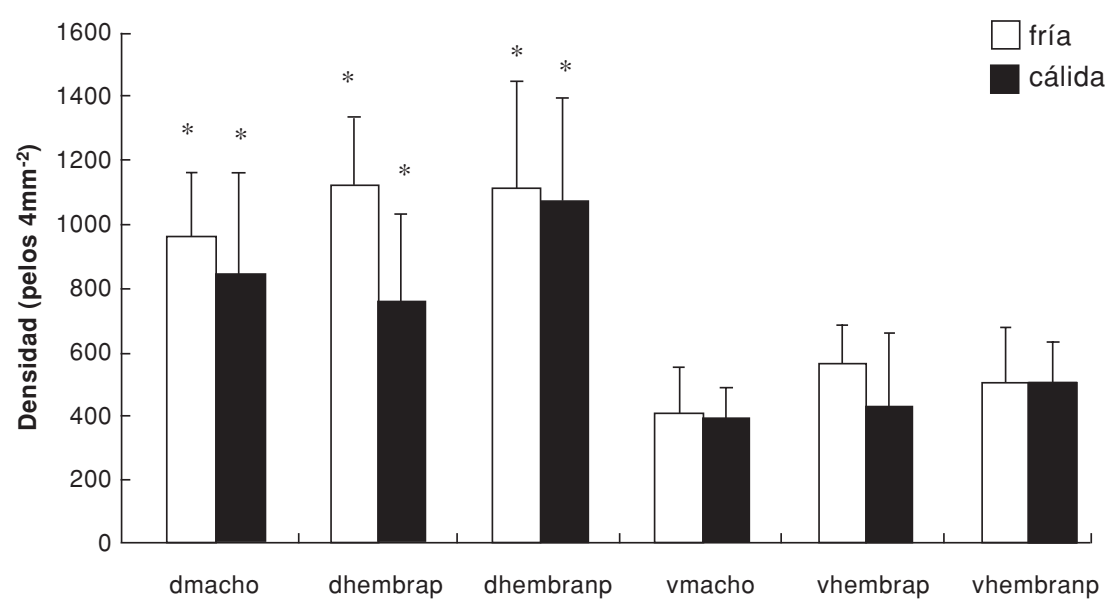

Región corporal, sexo y condición reproductiva

Fig. 1: Valores promedios de densidad de pelos observada en el dorso de machos (dmacho), dorso de hembras preñadas (dhembrap), dorso de hembras no preñadas (dhembranp), vientre de machos (vmacho), vientre de hembras preñadas (vhembrap) y vientre de hembras no preñadas (vhembranp) en las estaciones cálida y fría. (*) indica diferencias significativas en densidad entre el dorso y vientre para el mismo sexo/ condición reproductiva y estación del año.

Average fur density observed in males' back (dmacho), pregnant females' back (dhembrap), non-pregnant females' back (dhembranp), males' belly (vmacho), pregnant females' belly (vhembrap) and non-pregnant females' belly (vhembranp). (*) represents significant differences in fur density between back and belly for the same sex/ reproductive condition and season. 


\section{Longitud del pelaje}

No se encontró relación entre el peso de los individuos y la longitud del pelaje dorsal en la estación fría $\left(\mathrm{n}=27, \mathrm{r}^{2}=0,008 ; \mathrm{F}_{1,25}=0,21 ; \mathrm{P}=\right.$ $0,65)$ ni en la cálida $\left(\mathrm{n}=30, \mathrm{r}^{2}=0,094 ; \mathrm{F}_{1,28}=\right.$ 2,93; $\mathrm{P}=0,098)$. Tampoco se encontró relación entre el peso de los individuos y la longitud del pelaje ventral en la estación fría $\left(n=27, r^{2}=\right.$ 0,$\left.031 ; \mathrm{F}_{1,25}=0,8 ; \mathrm{P}=0,38\right)$ ni la cálida $(\mathrm{n}=30$, $\left.\mathrm{r}^{2}=0,0014, \mathrm{~F}_{1,28}=0,04 \mathrm{P}=0,85\right)$. Se observó efecto de la región corporal y de la estación del año sobre la longitud del pelaje, al igual que de la interacción entre región corporal, estación y sexo/condición reproductiva de las hembras (Tabla 3, Fig. 2). Tanto los machos como las hembras preñadas y no preñadas presentaron un pelaje dorsal significativamente más largo que el ventral en ambas estaciones (Tabla 3, Fig. 2). En la estacion fría, los machos, hembras preñadas y hembras no preñadas exhibieron un pelaje dorsal significativamente más largo respecto a la estación cálida (Tabla 3, Fig. 2). Por último, en la estación cálida, las hembras preñadas exhibieron un pelaje ventral significativamente más corto que el de machos y hembras no preñadas (Tukey, $\mathrm{P}_{1}=0,04 ; \mathrm{P}_{2}=0,035$ ).

\section{DISCUSIÓN}

El aislamiento térmico del pelaje depende tanto de la longitud como de la densidad de los pelos $\mathrm{y}$, en general, las especies que presentan alta densidad tienen pelos relativamente más cortos
(Spotorno et al. 1998). Modificaciones de las características del pelaje a lo largo del año han sido observadas en roedores de superficie (Hart \& Heroux 1953, Gorecki 1966, Khateeb \& Johnson 1971, Sealander 1972, Bolshakov 1984, Bozinovic et al. 1990, Bozinovic \& Merrit 1992), los cuales se encuentran expuestos a mayores variaciones de la temperatura ambiente que los roedores subterráneos; y en particular en Microtus ochrogaster, el aumento de la longitud del pelaje invernal estaría regulado por el fotoperíodo (Smale et al. 1988). A diferencia de otros roedores subterráneos, C. talarum forrajea en superficie, aventurándose fuera de los túneles por breves períodos para recolectar vegetación (Comparatore 1995, Busch et al. 2000, del Valle et al. 2001). Por lo tanto, aunque las temperaturas registradas durante este estudio en el suelo del interior de la cueva presentaron menor variación estacional que las temperaturas del aire de superficie, estos roedores no solo enfrentan la variación térmica del ambiente de la cueva sino que, por breves períodos de tiempo, también experimentan las temperaturas de superficie. En C. talarum, el cambio estacional de las características del pelaje representaría, entonces, un ajuste a la variación de las temperaturas en el interior y exterior de la cueva. Este ajuste parece involucrar principalmente cambios en la longitud y, en menor medida, en la densidad, ya que en este estudio se observaron diferencias significativas en la longitud del pelaje de esta especie entre la estación cálida y la fría, siendo mayor la longitud en este último período.

TABLA 3

Tabla de ANOVA de tres vías con el sexo/condición reproductiva de las hembras (machos, hembras preñadas y hembras no preñadas, 1) y la estación del año (cálida y fría, 2) como los factores "entre individuos" y la región corporal (dorso y vientre, 3 ) como el factor "en individuos" para evaluar su efecto el sobre la longitud del pelaje

Table of a three-way ANOVA, with sex/reproductive condition of the females (males, pregnant females and non-pregnant females, 1) and season of the year (warm and cold, 2) as between-subject effects and body region (back and belly, 3) as within- subject effect to evaluate their effect on fur length

\begin{tabular}{|c|c|c|c|c|}
\hline Factor & Grados de libertad & Cuadrado medio & Valor de F & Valor de $\mathrm{P}$ \\
\hline 1 & 2 & 1,724 & 0,631 & 0,536 \\
\hline 2 & 1 & 423,63 & 155,07 & $<0,0001$ \\
\hline 12 & 2 & 1,656 & 0,606 & 0,549 \\
\hline Error & 51 & 2,732 & & \\
\hline 3 & 1 & 321,95 & 132,28 & $<0,0001$ \\
\hline 31 & 2 & 1,553 & 0,638 & 0,532 \\
\hline 32 & 1 & 0,591 & 0,243 & 0,624 \\
\hline 123 & 2 & 9,256 & 3,803 & 0,029 \\
\hline Error & 51 & 2,434 & & \\
\hline
\end{tabular}




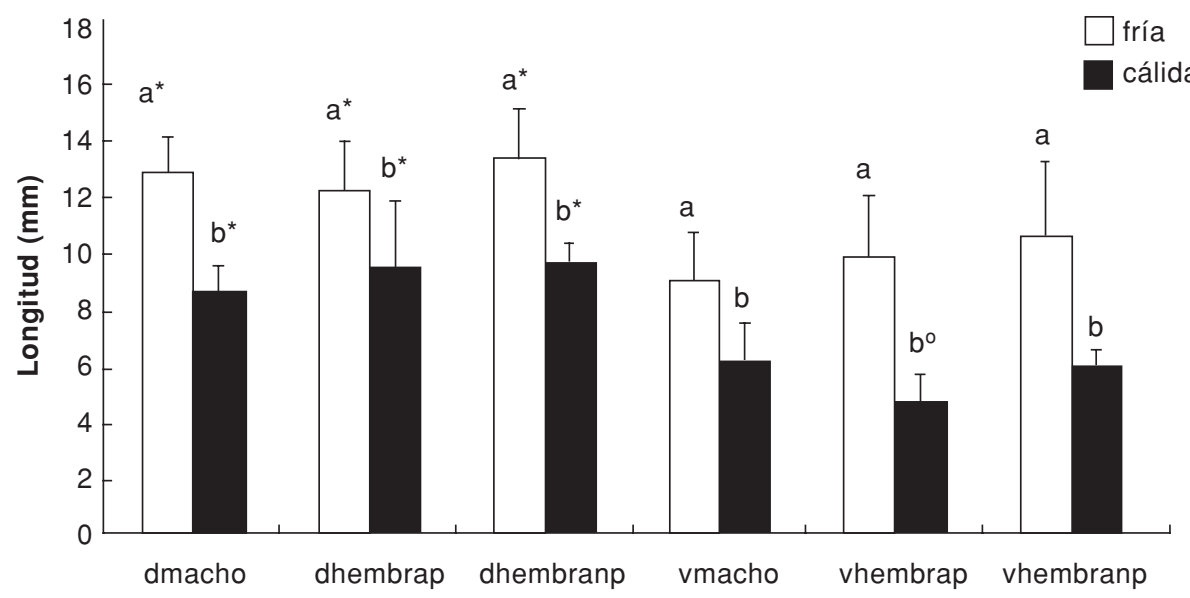

Región corporal, sexo y condición reproductiva

Fig. 2: Valores promedios de longitud de pelos observada en el dorso de machos (dmacho), dorso de hembras preñadas (dhembrap), dorso de hembras no preñadas (dhembranp), vientre de machos (vmacho), vientre de hembras preñadas (vhembrap) y vientre de hembras no preñadas (vhembranp). (*) indica diferencias significativas en longitud entre el dorso y vientre para el mismo sexo/condición reproductiva y estación del año. Letras diferentes indican diferencias significativas en longitud entre estaciones para el mismo sexo/condición reproductiva y región corporal. $\left({ }^{\circ}\right)$ representa diferencias significativas en longitud entre sexos y condición reproductiva de las hembras para una misma estación del año y región corporal.

Average fur length observed in males' back (dmacho), pregnant females' back (dhembrap), non-pregnant females' back (dhembranp), males' belly (vmacho), pregnant females' belly (vhembrap) and non-pregnant females' belly (vhembranp). $\left.{ }^{*}\right)$ represents significant differences in fur length between back and belly for the same sex/ reproductive condition and season. Different letters represent significant differences in length between seasons for the same sex/reproductive condition and body region. $\left({ }^{\circ}\right)$ represents significant differences in length between sexes and reproductive conditions of the females for the same season and body region.

El máximo intercambio de calor por conducción entre el vientre y el suelo se alcanza cuando los animales presionan su superficie ventral contra el sustrato, lo que ha sido descripto como regulación tigmotérmica (Buffenstein 2000). Este comportamiento ha sido observado en Heterocephalus glaber, que intenta ganar calor al presionar su vientre contra el sustrato de los túneles más cálidos cercanos a la superficie (Brett 1991, Buffenstein \& Yahav 1991). Asimismo, Luna \& Antinuchi (resultados no publicados) observaron que individuos de $C$. talarum cavando a temperaturas ambientales superiores al rango de termoneutralidad de la especie $\left(35^{\circ} \mathrm{C}\right)$ no sufrieron un aumento significativo de su temperatura corporal y su conductancia térmica se incrementó con respecto a la registrada a 15 y $25{ }^{\circ} \mathrm{C}$. Mientras excavaban y removían el suelo excavado, el vientre de estos roedores se encontraba en estrecho contacto con el sustrato. En este sentido, en el presente estudio el pelaje ventral de $C$. talarum resultó significativamente menos denso y menos largo que el del dorso, en cualquiera de las estaciones consideradas y en ambos sexos, sin importar la condición reproductiva de las hembras. Estas características del pelaje ventral de $C$. talarum, comunes también en otros grupos de mamíferos, incrementarían la conducción de calor desde el animal hacia el sustrato de la cueva, especialmente durante períodos de mayor actividad a temperaturas ambientales altas, cuando el riesgo de sobrecalentamiento sería mayor (ver McNab 1966, "hipótesis del stress térmico").

En trabajos previos, se ha propuesto que durante la preñez las hembras de mamíferos sufren cambios en el flujo de calor hacia el ambiente debidos principalmente a modificaciones de su tamaño, forma, tasa metabólica y cantidad de grasa corporal acumulada (Buffenstein 2000). A diferencia de las hembras de $H$. glaber, que exhiben un incremento de su tasa metabólica durante la preñez, las hembras preñadas de $C$. talarum mantienen una tasa metabólica similar a la de las hembras no preñadas y experimentan un incremento de su peso corporal de hasta un $41 \%$ (Zenuto et al. 2002a). Sin embargo, la temperatura corporal de las hembras preñadas no sufre aumentos marcados con respecto a la de hembras no reproductivas. 
En este estudio se observó que, en la estación cálida, las hembras preñadas exhibieron un pelaje ventral significativamente más corto que el pelaje ventral de machos y de hembras no preñadas. Debido a que el peso corporal, y por lo tanto el volumen, aumentan durante la preñez y la transferencia de calor hacia el ambiente por conducción podría reducirse, la estación cálida representaría mayores dificultades termorregulatorias para las hembras preñadas con respecto a las hembras no preñadas y machos. Por lo tanto, la reducción de la longitud del pelaje podría aumentar el flujo de calor corporal hacia el ambiente en períodos de altas temperaturas ambientales y contribuir a la normotermia de las hembras preñadas. Sin embargo, las modificaciones en características del pelaje, aunque de importancia, no constituirían el único mecanismo compensatorio de las hembras preñadas para afrontar períodos térmicamente estresantes. Se ha observado que las hembras preñadas presentan uñas significativamente más largas que las hembras no preñadas y machos (A.P. Cutrera, resultados no publicados), lo cual podría indicar una disminución del desgaste de estas vinculado a la reducción de la actividad excavatoria durante la preñez, como otra forma de evitar el sobrecalentamiento.

Por otro lado, se ha observado que las crías de $C$. talarum dependen en gran medida de la presencia de la madre para regular su temperatura corporal y permanecen en contacto con ella en el nido el $80 \%$ del tiempo hasta los 15 días de edad, momento en que comienzan a termorregular independientemente (Zenuto et al. 2002a, Zenuto et al. 2002b, Cutrera et al. 2003). El contacto entre madre y cría involucra la superficie ventral materna y la superficie dorsal de las crías, por lo que la presencia de pelos más cortos en el vientre de las hembras preñadas cercanas a la lactancia podría contribuir a la transferencia de calor a las crías a partir de su nacimiento. Sin embargo, dicha reducción de la longitud de los pelos del vientre de hembras preñadas se verificó en este estudio durante la estación cálida, en la cual el ambiente térmico de la cueva es favorable para las crías y donde la necesidad de recibir calor corporal por parte de la hembra no sería vital.

A pesar del rol obvio que cumple el pelaje en los procesos de transferencia de calor al ambiente, son escasos los estudios que han investigado las características del mismo como aislante térmico en roedores subterráneos y no existen hasta la fecha trabajos que hayan considerado los posibles cambios en sus propiedades durante períodos térmicamente estresantes, como es la preñez, en hembras de este grupo de micromamíferos.

En futuros estudios sería de interés explorar aspectos energéticos relacionados con la presencia y ausencia del pelaje y conocer cuáles son los efectos del mismo sobre la conductancia térmica de los individuos de C. talarum.

\section{AGRADECIMIENTOS}

Los autores de este trabajo desean agradecer a los miembros del Laboratorio de Ecofisiología por su apoyo constante; en especial al Lic. F. Luna y al Dr. M. Kittlein por la colaboración prestada en los análisis estadísticos. Agradecemos también los comentarios de dos revisores anónimos que contribuyeron a mejorar este manuscrito sustancialmente. Este trabajo fue financiado por la Universidad Nacional de Mar del Plata.

\section{LITERATURA CITADA}

BOLSHAKOV VN (1984) Winter ecology of small mammals in the Ural Mountains. En: Merritt JF (ed) Winter ecology of small mammals: 103-108. Special Publication, Carnegie Museum of Natural History 10, Pittsburgh, Pennsylvania, USA.

BOZINOVIC F \& JF MERRITT (1992) Summer and winter thermal conductance conductance of Blarina brevicauda (Mammalia: Insectivora: Soricidae) inhabiting the Appalachian Mountains. Annals of Carnegie Museum 61: 33-37.

BOZINOVIC F, FF NOVOA \& C VELOSO (1990) Seasonal changes in energy expenditure and digestive tract of Abrothrix andinus (Cricetidae) in the Andes range. Physiological Zoology 63: 12161231.

BUFFENSTEIN R (2000) Ecophysiological responses of subterranean rodents to underground habitats. En: Lacey EA, JL Patton \& GN Cameron (eds) Life Underground: 62-110. The University of Chicago Press, Chicago, Illinois, USA.

BRETT RA (1991) The ecology of naked mole-rat colonies: burrowing food and limiting factors. En: Sherman PW, JUM Jarvis \& RD Alexander (eds) The biology of the naked mole-rat: 137-184. Princeton University Press, Princeton, New Jersey, USA.

BUFFENSTEIN R \& S YAHAV (1991) Is the naked molerat, Heterocephalus glaber, a poikilotermic or poorly thermoregulating endothermic mammal? Journal of Thermal Biology 16: 227-232.

BUSCH C, CD ANTINUCHI, JC DEL VALLE, MJ KITTLEIN, AI MALIZIA, AI VASSALLO \& RR ZENUTO (2000). En: Lacey EA, JL Patton \& GN Cameron (eds) Life Underground: 183-225. The University of Chicago Press, Chicago, Illinois, USA.

COMPARATORE VM, MS CID \& C BUSCH (1995) Dietary preferences of two sympatric subterranean rodent populations in Argentina. Revista Chilena de Historia Natural 68: 197-206. 
COSSINS AR \& K BOWLER (1987) Temperature biology of animals. Chapman \& Hall, London, United Kingdom. 339 pp.

CUTRERA AP, CD ANTINUCHI \& C BUSCH (2003) Thermoregulatory development in pups of the subterranean rodent Ctenomys talarum. Physiology and Behavior 79: 321-330.

DEL VALLE JC, MI LOHFELT, VM COMPARATORE, MS CID \& C BUSCH (2001) Feeding selectivity and food preference of Ctenomys talarum (tucotuco). Mammalian Biology 66: 165-173.

GORECKI A (1966) Metabolic acclimation of bank voles to laboratory conditions. Acta Theriologica 11: 399-407.

HART JS \& O HEROUX (1953) A comparison of some seasonal and temperature-induced changes in Peromyscus: cold resistance, metabolism and pelage insulation. Canadian Journal of Zoology 63: 1216-1231.

KHATEEB A \& E JOHNSON (1971) Seasonal changes of pelage in the vole (Microtus agrestis). I. Correlation with changes in the endocrine glands. General and Comparative Endocrinology 16: 217-228.

KLAUER G, H BURDA \& E NEVO (1997) Adaptive differentiations of the skin of the head in a subterranean rodent, Spalax ehrenbergi. Journal of Morphology 233: 53-66.

KRUPA JJ \& KN GELUSO (2000) Matching the color of excavated soil: cryptic coloration in the plains pocket gopher (Geomys bursarius). Journal of Mammalogy 81: 86-96.
McNAB BK (1966) The metabolism of fossorial rodents: A study of convergence. Ecology 47: 712-733.

McNAB BK (1979) The influence of body size on the energetics and distribution of fossorial and burrowing mammals. Ecology 60: 1010-1021.

SEALANDER JA (1972) Circum-annual changes in age, pelage characteristics and adipose tissue in the northern red-backed vole in interior Alaska. Acta Theriologica 17: 1-24.

SMALE L, RJ NELSON \& I ZUCKER (1988) Daylength influences pelage and plasma prolactin concentrations but not reproduction in the prairie vole, Microtus ochrogaster. Journal of Reproduction and Fertility 83: 99-106.

SPOTORNO AE, C ZULETA, A GANTZ, F SAIZ, J RAU, M ROSENMAN, A CORTÉS, G RUIZ, L YATES, E COUVE \& JC MARÍN (1998) Sistemática y adaptación de mamíferos, aves e insectos fitófagos de la región de Antofagasta, Chile. Revista Chilena de Historia Natural 71: 501-526.

ZENUTO RR, CA ANTINUCHI \& C BUSCH (2002a) Bioenergetics of reproduction and pup development in a subterranean rodent (Ctenomys talarum). Physiological and Biochemical Zoology 75: 469-478.

ZENUTO RR, AI VASSALLO \& C BUSCH (2002b) Comportamiento social y reproductivo del roedor subterráneo solitario Ctenomys talarum (Rodentia: Ctenomyidae) en condiciones de semicautiverio. Revista Chilena de Historia Natural 75: 165-177. 